\title{
SPACE-LIKE ISOTHERMIC SURFACES AND GRASSMANNIAN SYSTEMS
}

\author{
By \\ Martha P. Dussan*
}

\begin{abstract}
We show that space-like isothermic surfaces in the pseudo-riemannian space $\mathbf{R}^{n-j, j}$ are associated to $O(n-j+1, j+1) /$ $O(n-j, j) \times O(1,1)$-system, and that the action of a rational map with two simple poles on the space of local solutions of $O(n-j+1, j+1) / O(n-j, j) \times O(1,1)$-system correspond to Ribaucour and Darboux transformations to space-like surfaces in $\mathbf{R}^{n-j, j}$.
\end{abstract}

\section{Introduction}

It is known that there is a connection between the theory of submanifolds and the theory of solitons. Some examples are the well-known local correspondence between pseudospherical surface and the solutions of the Sine-Gordon equation $q_{x t}=\sin q$, and the recent reformulation of the theory of isothermic surfaces in $\mathbf{R}^{3}$ within the modern theory of completely integrable (soliton) systems, given in [4]. A key point to study this connection is the existence of a Lax Pair or a zero curvature representation which may give rise to an action of an infinite dimensional group on the space of local solutions of the equation, called the "dressing action" in the theory of soliton equations.

There are several excellent articles where is study that connection, specially in a recent work of Terng et al. ([9], [2]) is established a relationship between integrable systems and submanifolds geometry. In those articles it is considered a new integrable system the $U / K$-system and study the geometry associated to the particular $G_{m, n}=O(m+n) / O(m) \times O(n)$ and $G_{m, n}^{1}=O(m+n, 1) / O(m) \times$ $O(n, 1)$-system. This study involved to find submanifolds in a certain symmetric space whose Gauss-Codazzi-Ricci equations are given by these systems, as well as

* Author's e-mail: dussan@ime.usp.br

Key Words: $U / K$-system, Dressing action, Space-like immersion.

$\operatorname{AMS}(2000): 53 \mathrm{~A} 05,35 \mathrm{Q} 51$.

Received September 24, 2004.

Revised October 17, 2005. 
the geometric transformations associated to the dressing action of certain simple elements.

The $U / K$-system is defined by Terng in ([9]) as the following PDE: Let $U$ be a semi-simple Lie group, $\sigma$ an involution on $U$ and $K$ the fixed point set of $\sigma$. Then $U / K$ is a symmetric space. The Lie algebra $\mathscr{K}$ is fixed point set of the differential $\sigma_{*}$ of $\sigma$ at the identity, in others words, it is the +1 eigenspace of $\sigma_{*}$. Let $\mathscr{P}$ denote the -1 eigenspace of $\sigma_{*}$. Then we have $\mathscr{U}=\mathscr{K} \oplus \mathscr{P}$ and

$$
[\mathscr{K}, \mathscr{K}] \subset \mathscr{K}, \quad[\mathscr{K}, \mathscr{P}] \subset \mathscr{P}, \quad[\mathscr{P}, \mathscr{P}] \subset \mathscr{K} .
$$

Let $\mathscr{A}$ be a maximal abelian subalgebra in $\mathscr{P}, a_{1}, a_{2}, \ldots, a_{n}$ a basis for $\mathscr{A}$ and $\mathscr{A}^{\perp}$ the orthogonal complement of $\mathscr{A}$ in the algebra $\mathscr{U}$ with respect to the Killing form $\langle$,$\rangle . The U / K$-system is the following first order non-linear PDE for $v: \mathbf{R}^{n} \rightarrow \mathscr{P} \cap \mathscr{A}^{\perp}$

$$
\left[a_{i}, v_{x_{j}}\right]-\left[a_{j}, v_{x_{i}}\right]=\left[\left[a_{i}, v\right],\left[a_{j}, v\right]\right], \quad 1 \leq i \neq j \leq n,
$$

where $v_{x_{j}}=\frac{\partial v}{\partial x_{j}}$. It is not difficult to show that $v$ is a solution of the $U / K$-system if and only if the connection 1 -form

$$
\theta_{\lambda}=\sum\left(a_{i} \lambda+\left[a_{i}, v\right]\right) d x_{i}
$$

is flat for all $\lambda \in \mathbf{C}$, if and only if there exists an application $E$ such that $E^{-1} d E=\theta_{\lambda}$. If $\theta_{\lambda}$ is flat for all $\lambda \in \mathbf{C}$ the $\theta_{0}=\sum_{i}\left[a_{i}, v\right] d x_{i}$ is a $\mathscr{K}$-valued, flat connection and hence there exists $g: \mathbf{R}^{n} \rightarrow K$ such that $g^{-1} d g=\theta_{0}$. Suppose $K=K_{1} \times K_{2}$ so we can write $g=\left(g_{1}, g_{2}\right) \in K_{1} \times K_{2}$. The two new systems given by the flatness of the gauge transformation $g_{1} * \theta_{\lambda}$ and $g_{2} * \theta_{\lambda}$ are called the $U / K$-system I (II resp.).

In the study made in ([2]), it was obtained that the submanifolds geometries associated to the $G_{m, n}$ and $G_{m, n}^{1}$-systems, include submanifolds in space forms with constant sectional curvatures, submanifolds admitting principal curvature coordinates and isothermic surfaces in $\mathbf{R}^{n}$. Moreover, that the dressing action of simple elements on the space of solutions of these systems correspond to Backlund, Darboux and Ribaucour transformations for submanifolds.

In this note we are interested in to discuss the geometry of surfaces associated to the $O(n-j+1, j+1) / O(n-j, j) \times O(1,1)$-system as well as the geometric transformations corresponding to dressing actions. We show that in this case, the space-like isothermic surfaces in the pseudo-riemannian space $\mathbf{R}^{n-j, j}$ for any signature $j$ are associated to $O(n-j+1, j+1) / O(n-j, j) \times O(1,1)$-system II, and that the Ribaucour and Darboux transformations to space-like surfaces 
correspond to the action of a rational map with two simples poles. In particular we obtain that the space-like 2-tuples in $\mathbf{R}^{n-j, j}$ of type $O(1,1)$ are in correspondence with the solutions of the $O(n-j+1, j+1) / O(n-j, j) \times O(1,1)$ system II, and that these correspond to an isothermic pair of space-like surfaces in $\mathbf{R}^{n-j, j}$. Some results of this note appeared initially in the research report No. 60 in 2002, see ([7]).

We should recall that the topic of isothermic surfaces has been of increasing interest to geometers because they can be reformulated within the soliton theory ([4]), or can be interpreted as the so-called curved flats in the symmetric space $O(4,1) / O(3) \times O(1,1)([3])$, and because of the relation between a 2-tuple in $\mathbf{R}^{3}$ of type $O(1,1)$ and an isothermic pair ([2]). So motivated by these relations and the general results in $\mathbf{R}^{n}([1])$, this note pay attention to the space-like isothermic surfaces and its relation with integrable systems. Finally, we observe that as in the classic situation, space-like minimal surfaces, space-like surfaces with constant mean curvature and space-like surfaces of revolution in $\mathbf{R}^{2,1}$, provide examples of space-like isothermic surfaces in the Lorenztian space ([8]).

\section{The associated geometry}

First of all, we will find one maximal abelian subalgebra in the subspace $\mathscr{P}$ for which we obtain elliptic Gauss equations, which is correct for space-like surfaces:

Let $U / K=O(n-j+1, j+1) / O(n-j, j) \times O(1,1), n-j \geq 2$, where

$$
\begin{gathered}
O(n-j+1, j+1)=\left\{X \in G L(n+2) \mid X^{t}\left(\begin{array}{cc}
I_{n-j, j} & 0 \\
0 & I_{1,1}
\end{array}\right) X=\left(\begin{array}{cc}
I_{n-j, j} & 0 \\
0 & I_{1,1}
\end{array}\right)\right\}, \\
I_{n-j, j}=\left(\begin{array}{cc}
I_{n-j} & 0 \\
0 & -I_{j}
\end{array}\right), \quad I_{1,1}=\left(\begin{array}{cc}
1 & 0 \\
0 & -1
\end{array}\right) .
\end{gathered}
$$

So, $O(n-j+1, j+1)$ is the Lie group of linear isomorphisms that leaves the following bilinear form on $\mathbf{R}^{n+2}$ invariant:

$$
\begin{aligned}
\langle x, y\rangle= & x_{1} y_{1}+x_{2} y_{2}+\cdots+x_{n-j} y_{n-j}-x_{n-j+1} y_{n-j+1} \\
& -\cdots-x_{n} y_{n}+x_{n+1} y_{n+1}-x_{n+2} y_{n+2} .
\end{aligned}
$$

Let $\mathscr{U}=o(n-j+1, j+1)$ be the Lie algebra of $U$ and $\sigma: \mathscr{U} \rightarrow \mathscr{U}$ be an involution defined by $\sigma(X)=I_{n, 2}^{-1} X I_{n, 2}$. Denote by $\mathscr{K}, \mathscr{P}$ the $+1,-1$ eigenspaces of $\sigma$ respectively, i.e., 


$$
\begin{gathered}
\mathscr{K}=\left\{\left(\begin{array}{cc}
Y_{1} & 0 \\
0 & Y_{2}
\end{array}\right) \mid Y_{1} \in o(n-j, j), Y_{2} \in o(1,1)\right\}=o(n-j, j) \times o(1,1), \\
\mathscr{P}=\left\{\left(\begin{array}{cc}
0 & \xi \\
-I_{1,1} \xi^{t} I_{n-j, j} & 0
\end{array}\right) \mid \xi \in \mathscr{M}_{n \times 2}\right\} .
\end{gathered}
$$

We define the matrices $a_{1}, a_{2} \in \mathscr{M}_{(n+2) \times(n+2)}$, by

$$
a_{1}=-e_{1, n+1}+e_{n+1,1}, \quad a_{2}=e_{2, n+2}+e_{n+2,2},
$$

where $e_{i j}$ is the elementary $(n+2) \times(n+2)$ matrix, whose only non-zero entry is 1 in the $i j^{\text {th }}$ place.

It is easy to see that the subalgebra $\mathscr{A}=\left\langle a_{1}, a_{2}\right\rangle$ is maximal abelian in $\mathscr{P}$. Then using this basis $\left\{a_{1}, a_{2}\right\}$, the $U / K$-system (1) for this symmetric space is the following PDE for

$$
\begin{gathered}
\xi=\left(\begin{array}{cc}
0 & \xi_{1} \\
\xi_{2} & 0 \\
r_{1,1} & r_{1,2} \\
\vdots & \vdots \\
r_{n-2,1} & r_{n-2,2}
\end{array}\right): \mathbf{R}^{2} \rightarrow \mathscr{M}_{n \times 2}, \\
\begin{cases}\left(r_{i, 2}\right)_{x_{1}}=r_{i, 1} \xi_{1}, \quad i=1, \ldots, n-2 \\
\left(r_{i, 1}\right)_{x_{2}}=r_{i, 2} \xi_{2}, \quad i=1, \ldots, n-2 \\
\left(\xi_{1}\right)_{x_{1}}+\left(\xi_{2}\right)_{x_{2}}=-\sum_{i=1}^{n-j-2} r_{i, 1} r_{i, 2}+\sum_{i=n-j-1}^{n-2} r_{i, 1} r_{i, 2}, \\
\left(\xi_{2}\right)_{x_{1}}=\left(\xi_{1}\right)_{x_{2}} .\end{cases}
\end{gathered}
$$

We now denote the entries of $\xi$ by:

$$
\left(\begin{array}{cc}
0 & \xi_{1} \\
\xi_{2} & 0
\end{array}\right)=F \quad \text { and } \quad\left(\begin{array}{cc}
r_{1,1} & r_{1,2} \\
\vdots & \vdots \\
r_{n-2,1} & r_{n-2,2}
\end{array}\right)=G
$$

The $O(n-j+1, j+1) / O(n-j, j) \times O(1,1)$-system II is the PDE for $(F, G, B): \mathbf{R}^{2} \rightarrow g l_{*}(2) \times \mathscr{M}_{(n-2) \times 2} \times O(1,1)$, where $g l_{*}(2)$ is the set of matrices $2 \times 2$ with diagonal elements zero,

$$
\left\{\begin{array}{l}
\left(r_{i, 2}\right)_{x_{1}}=r_{i, 1} \xi_{1}, \quad i=1, \ldots, n-2 \\
\left(r_{i, 1}\right)_{x_{2}}=r_{i, 2} \xi_{2}, \quad i=1, \ldots, n-2 \\
\left(\xi_{1}\right)_{x_{1}}+\left(\xi_{2}\right)_{x_{2}}=-\sum_{i=1}^{n-j-2} r_{i, 1} r_{i, 2}+\sum_{i=n-j-1}^{n-2} r_{i, 1} r_{i, 2} \\
\left(b_{i j}\right)_{x_{k}}=\xi_{k} b_{i k}, \quad k \neq j,
\end{array}\right.
$$


where the matrix $B=\left(b_{i j}\right) \in O(1,1)$. Now we take $B=\left(\begin{array}{cc}\cosh u & \sinh u \\ \sinh u & \cosh u\end{array}\right)$, and use the fact that

$$
B^{-1} d B=\left(\begin{array}{cc}
0 & \xi_{1} d x_{1}+\xi_{2} d x_{2} \\
\xi_{1} d x_{1}+\xi_{2} d x_{2} & 0
\end{array}\right),
$$

to have $\xi_{1}=u_{x_{1}}, \quad \xi_{2}=u_{x_{2}}$ and that $O(n-j+1, j+1) / O(n-j, j) \times O(1,1)$ system II becomes in the set of partial differential equations for $\left(u, r_{1,1}, r_{1,2}, \ldots, r_{n-2,1}, r_{n-2,2}\right)$ :

$$
\left\{\begin{array}{l}
\left(r_{i, 2}\right)_{x_{1}}=r_{i, 1} u_{x_{1}}, \quad i=1, \ldots, n-2 \\
\left(r_{i, 1}\right)_{x_{2}}=r_{i, 2} u_{x_{2}}, \quad i=1, \ldots, n-2 \\
\left(u_{x_{1}}\right)_{x_{1}}+\left(u_{x_{2}}\right)_{x_{2}}=-\sum_{i=1}^{n-j-2} r_{i, 1} r_{i, 2}+\sum_{i=n-j-1}^{n-2} r_{i, 1} r_{i, 2} .
\end{array}\right.
$$

Next we identify the geometries of space-like surfaces corresponding to the $O(n-j+1, j+1) / O(n-j, j) \times O(1,1)$-system II. We start by defining the spacelike 2-tuples in the pseudo-riemannian space $\mathbf{R}^{n-j, j}$ of type $O(1,1)$ :

Definition 2.1. Let $\mathcal{O}$ be a domain in $\mathbf{R}^{2}$ and $X_{i}: \mathcal{O} \rightarrow \mathbf{R}^{n-j, j}$ an immersion with flat and non-degenerate normal bundle for $i=1,2 .\left(X_{1}, X_{2}\right)$ is called a spacelike 2-tuple in $\mathbf{R}^{n-j, j}$ of type $O(1,1)$ if:

(i) The normal plane of $X_{1}(x)$ is parallel to the normal plane of $X_{2}(x)$ for any $x \in \mathcal{O}$,

(ii) there exists a common parallel normal frame $\left\{e_{3}, \ldots, e_{n}\right\}$, where $\left\{e_{\alpha}\right\}_{3}^{n-j}$ and $\left\{e_{\alpha}\right\}_{n-j+1}^{n}$ are space-like and time-like vectors resp.

(iii) $x \in \mathcal{O}$ is a hyperbolic line of curvature coordinate system with respect to $\left\{e_{3}, \ldots, e_{n}\right\}$ for each $X_{k}$ such that the fundamental forms of $X_{k}$ are:

$$
\begin{gathered}
I_{k}=b_{k 1}^{2} d x_{1}^{2}+b_{k 2}^{2} d x_{2}^{2}, \\
I I_{k}=\varepsilon_{k} \sum_{i=1}^{n-2}\left(b_{k 1} g_{i 1} d x_{1}^{2}+b_{k 2} g_{i 2} d x_{2}^{2}\right) e_{i+2}, \quad \varepsilon_{1}=-\varepsilon_{2}=1,
\end{gathered}
$$

for some $O(1,1)$-valued map $B=\left(b_{i j}\right)$ and a $\mathscr{M}_{(n-2) \times 2}$-valued map $G=\left(g_{i j}\right)$.

Our first result, which gives us the relationship between the space-like 2-tuples and the solutions of the $O(n-j+1, j+1) / O(n-j, j) \times O(1,1)$-system II (5), is the following:

THEOREM 2.1. Suppose $\left(u, r_{1,1}, r_{1,2}, \ldots, r_{n-2,1}, r_{n-2,2}\right)$ is solution of $(5)$ and $F$, $B$ are given by 


$$
F=\left(\begin{array}{cc}
0 & \xi_{1} \\
\xi_{2} & 0
\end{array}\right)=\left(\begin{array}{cc}
0 & u_{x_{1}} \\
u_{x_{2}} & 0
\end{array}\right), \quad B=\left(\begin{array}{cc}
\cosh u & \sinh u \\
\sinh u & \cosh u
\end{array}\right)
$$

Then: $(a)$

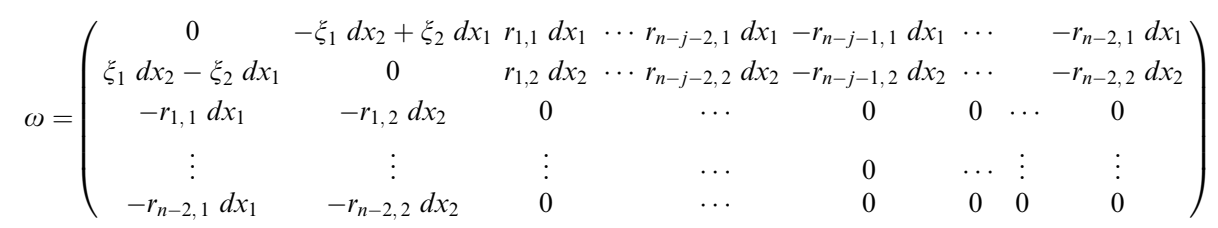

is a flat $o(n-j, j)$-valued connection 1-form. Hence there exists $A: \mathbf{R}^{2} \rightarrow$ $O(n-j, j)$ such that

$$
A^{-1} d A=\omega
$$

where $\omega$ is given by (7).

(b) $A\left(\begin{array}{cccccc}-d x_{1} & 0 & 0 & \cdots & 0 & 0 \\ 0 & d x_{2} & 0 & \cdots & 0 & 0\end{array}\right)^{t} B^{-1}$ is exact. So there exists a map $X: \mathbf{R}^{2} \rightarrow \mathscr{M}_{n \times 2}$ such that

$$
d X=A\left(\begin{array}{cccccc}
-d x_{1} & 0 & 0 & \cdots & 0 & 0 \\
0 & d x_{2} & 0 & \cdots & 0 & 0
\end{array}\right)^{t} B^{-1}
$$

(c) Suppose that all the entries of $B$ are non-zero. Let $X_{j}: \mathbf{R}^{2} \rightarrow \mathbf{R}^{n-j, j}$ denote the j-th column of $X$ (solution of $(9))$ and $e_{i}$ denotes the $i$-th column of $A$. Then $\left(X_{1}, X_{2}\right)$ is a space-like 2-tuple in $\mathbf{R}^{n-j, j}$ of type $O(1,1)$. In fact,

(1) $e_{1}, e_{2}$ are space-like tangent vectors to $X_{1}$ and $X_{2}$, i.e., the tangent planes of $X_{1}, X_{2}$ are parallel.

(2) $\left\{e_{3}, \ldots, e_{n}\right\}$ is a parallel normal frame for $X_{1}$ and $X_{2}$, with $\left\{e_{3}, \ldots, e_{n-j}\right\}$ and $\left\{e_{n-j+1}, \ldots, e_{n}\right\}$ being resp. space-like and time-like vectors.

(3) the two fundamental forms for the immersions $X_{k}$ are:

$$
\left\{\begin{array}{l}
I_{1}=\cosh ^{2} u d x_{1}^{2}+\sinh ^{2} u d x_{2}^{2} \\
I_{1}=\sum_{i=1}^{n-2}\left(r_{i, 1} \cosh u d x_{1}^{2}+r_{i, 2} \sinh u d x_{2}^{2}\right) e_{i+2} \\
I_{2}=\sinh ^{2} u d x_{1}^{2}+\cosh ^{2} u d x_{2}^{2} \\
I_{2}=-\sum_{i=1}^{n-2}\left(r_{i, 1} \sinh u d x_{1}^{2}+r_{i, 2} \cosh u d x_{2}^{2}\right) e_{i+2}
\end{array}\right.
$$

Proof OF THEOREM 2.1. The proof follows from an argument similar to those for Theorem 6.8 or 7.4 in [2].

REMARK 2.1. We observe that, taking a generic $b=\left(b_{i j}\right) \in O(1,1)$, Theorem (2.1) shows that $I_{j}, I I_{j}$ are given by (6) and $d X_{j}$ are: 


$$
d X_{1}=-\left(b_{11} d x_{1} e_{1}+b_{12} d x_{2} e_{2}\right) \quad d X_{2}=\left(b_{21} d x_{1} e_{1}+b_{22} d x_{2} e_{2}\right)
$$

We also note that Theorem (2.1) can be stated for a generic $(F, G, B)$ solution of the system (4).

Now we have the converse theorem.

THEOREM 2.2. Let $\left(X_{1}, X_{2}\right)$ be a space-like 2-tuple in $\mathbf{R}^{n-j, j}$ of type $O(1,1)$, $\left\{e_{3}, \ldots, e_{n}\right\}$ a common parallel normal frame and $\left(x_{1}, x_{2}\right)$ a common hyperbolic line of curvature coordinates for $X_{1}$ and $X_{2}$, such that the two fundamental forms $I_{k}, I I_{k}$ for $X_{k}$ are given by (6). Set $f_{i j}=\frac{\left(b_{1 j}\right)_{x_{i}}}{b_{1 i}}$ if $i \neq j, f_{i i}=0$ and $F=\left(f_{i j}\right)_{2 \times 2}$. If all entries of $G$ are non-zero then $(F, G, B)$ is a solution of (4).

Proof. From the definition of space-like 2-tuples in $\mathbf{R}^{n-j, j}$, we have

$$
\omega_{1}^{(k)}=-\varepsilon_{k} b_{k 1} d x_{1}, \quad \omega_{2}^{(k)}=-\varepsilon_{k} b_{k 2} d x_{2}
$$

is a dual 1-frame for $X_{k}$ and $\omega_{1 \alpha}^{(k)}=\sigma_{\alpha} r_{\alpha-2,1} d x_{1}, \omega_{2 \alpha}^{(k)}=\sigma_{\alpha} r_{\alpha-2,2} d x_{2}$ for each $X_{k}$, where $\sigma_{\alpha}=1$ if $\alpha=3, \ldots, n-j$ and $\sigma_{\alpha}=-1$ if $\alpha=n-j+1, \ldots, n$. We observe that $\omega_{i \alpha}^{(k)}, i=1,2, \alpha=3, \ldots, n$ are independent of $k$. We have the Levi-Civita connection 1 -form for the metric $I_{k}$ is:

$$
\omega_{12}^{(k)}=\frac{\left(b_{k 1}\right)_{x_{2}}}{b_{k 2}} d x_{1}-\frac{\left(b_{k 2}\right)_{x_{1}}}{b_{k 1}} d x_{2}=f_{21}^{(k)} d x_{1}-f_{12}^{(k)} d x_{2}
$$

where $f_{21}^{(k)}=\frac{\left(b_{k 1}\right)_{x_{2}}}{b_{k 2}}, \quad f_{12}^{(k)}=\frac{\left(b_{k 2}\right)_{x_{1}}}{b_{k 1}}$. Since $d \omega_{1 \alpha}^{(k)}=-\omega_{12}^{(k)} \wedge \omega_{2 \alpha}^{(k)} \quad$ and $\quad d \omega_{2 \alpha}^{(k)}=$ $-\omega_{21}^{(k)} \wedge \omega_{1 \alpha}^{(k)}$ (see appendix), we get

$$
\frac{\left(b_{k 2}\right)_{x_{1}}}{b_{k 1}}=\frac{\left(r_{\alpha-2,2}\right)_{x_{1}}}{r_{\alpha-2,1}}, \quad \frac{\left(b_{k 1}\right)_{x_{2}}}{b_{k 2}}=\frac{\left(r_{\alpha-2,1}\right)_{x_{2}}}{r_{\alpha-2,2}},
$$

so $f_{21}^{(k)}, f_{12}^{(k)}$ are independent of $k$. Hence $\omega_{12}^{(k)}=\omega_{12}^{(1)}=\frac{\left(b_{11}\right)_{x_{2}}}{b_{12}} d x_{1}-\frac{\left(b_{12}\right)_{x_{1}}}{b_{11}} d x_{2}=$ $f_{21} d x_{1}-f_{12} d x_{2}=\xi_{2} d x_{1}-\xi_{1} d x_{2}$. So the structure equations and the GaussCodazzi equations for $X_{1}, X_{2}$ imply that $(F, G, B)$ is a solution of system (4).

THEOREM 2.3. The $O(n-j+1, j+1) / O(n-j, j) \times O(1,1)$-system II (4) is the Gauss-Codazzi equations for a space-like surface in $\mathbf{R}^{n-j, j}$ such that:

$$
I=\sinh ^{2} u d x_{1}^{2}+\cosh ^{2} u d x_{2}^{2}, \quad I I=-\sum_{i=1}^{n-2}\left(r_{i, 1} \sinh u d x_{1}^{2}+r_{i, 2} \cosh u d x_{2}^{2}\right) e_{i+2}
$$


Proof. From the form of I and II, we have:

$\omega_{1}=\sinh u d x_{1}, \quad \omega_{2}=\cosh u d x_{2}, \quad \omega_{1 \alpha}=\sigma_{\alpha} r_{\alpha-2,1} d x_{1}, \quad \omega_{2 \alpha}=\sigma_{\alpha} r_{\alpha-2,2} d x_{2}$, where $\sigma_{\alpha}=1$ if $\alpha=3, \ldots, n-j$ and $\sigma_{\alpha}=-1$ if $\alpha=n-j+1, \ldots, n$. Now use the structure equation to obtain: $\omega_{12}=u_{x_{2}} d x_{1}-u_{x_{1}} d x_{2}$. Using the Gauss-Codazzi equation (see appendix), we obtain that these are the following system for $\left(u, r_{1,1}, r_{1,2}, \ldots, r_{n-2,1}, r_{n-2,2}\right)$ :

$$
\left\{\begin{array}{l}
\left(r_{i, 2}\right)_{x_{1}}=r_{i, 1} u_{x_{1}}, \quad i=1, \ldots, n-2 \\
\left(r_{i, 1}\right)_{x_{2}}=r_{i, 2} u_{x_{2}}, \quad i=1, \ldots, n-2 \\
u_{x_{1} x_{1}}+u_{x_{2} x_{2}}=-\sum_{i=1}^{n-j-2} r_{i, 1} r_{i, 2}+\sum_{i=n-j-1}^{n-2} r_{i, 1} r_{i, 2} .
\end{array}\right.
$$

Hence if we put

$$
B=\left(\begin{array}{cc}
\cosh u & \sinh u \\
\sinh u & \cosh u
\end{array}\right), \quad F=\left(\begin{array}{cc}
0 & u_{x_{1}} \\
u_{x_{2}} & 0
\end{array}\right), \quad G=\left(\begin{array}{cc}
r_{1,1} & r_{1,2} \\
\vdots & \vdots \\
r_{n-2,1} & r_{n-2,2}
\end{array}\right),
$$

we see $(F, G, B)$ is solution of the system (4). Conversely, if $(F, G, B)$ is solution of the system (4), and we assume $B$ is as in (11), then from the fourth equation of system (4) we get $\xi_{1}=u_{x_{1}}, \xi_{2}=u_{x_{2}}$, i.e., $(F, G, B)$ is of the form (11). Finally writing the $O(n-j+1, j+1) / O(n-j, j) \times O(1,1)$-system II for this $(F, G, B)$, in terms of $u$ and $r_{i j}$ we get equation (10).

Combining Theorems 2.1 and 2.3 we get

THEOREM 2.4. Let $\mathcal{O}$ be a domain of $\mathbf{R}^{2}$, and $X_{2}: \mathcal{O} \rightarrow \mathbf{R}^{n-j, j}$ an immersion with flat normal bundle and $(x, y) \in \mathcal{O}$ a hyperbolic line of curvature coordinates system with respect to a parallel normal frame $\left\{e_{3}, \ldots, e_{n}\right\}$. Then there exists an immersion $X_{1}$, unique up to translation, such that $\left(X_{1}, X_{2}\right)$ is a space-like 2-tuple in $\mathbf{R}^{n-j, j}$ of type $O(1,1)$. Moreover, the fundamental forms of $X_{1}, X_{2}$ are respectively:

$$
\left\{\begin{array}{l}
I_{1}=\cosh ^{2} u d x_{1}^{2}+\sinh ^{2} u d x_{2}^{2} \\
I_{1}=\sum_{i=1}^{n-2}\left(r_{i, 1} \cosh u d x_{1}^{2}+r_{i, 2} \sinh u d x_{2}^{2}\right) e_{i+2} \\
I_{2}=\sinh ^{2} u d x_{1}^{2}+\cosh ^{2} u d x_{2}^{2} \\
I_{2}=-\sum_{i=1}^{n-2}\left(r_{i, 1} \sinh u d x_{1}^{2}+r_{i, 2} \cosh u d x_{2}^{2}\right) e_{i+2}
\end{array}\right.
$$

EXAMPLE 2.1. Recall that given a space-like surface in $\mathbf{R}^{2,1}$ with curvature -1 and free of umbilic points, there exists a local coordinates system $x_{1}, x_{2}$ such that the two fundamental forms are ([11]): 


$$
I=\cosh ^{2} u d x_{1}^{2}+\sinh ^{2} u d x_{2}^{2}, \quad I I=\cosh u \sinh u\left(d x_{1}^{2}+d x_{2}^{2}\right) .
$$

With respect to this coordinates system, the Gauss-Codazzi equation of the surface is written in the following form (Elliptic sinh-Gordon equation):

$$
u_{x_{1} x_{1}}+u_{x_{2} x_{2}}=\sinh u \cosh u
$$

This implies that $(u, \sinh u, \cosh u)$ is a solution of $O(3,2) / O(2,1) \times O(1,1)$-system $I I(5)$. Let $X\left(x_{1}, x_{2}\right)$ denote the immersion of $M$ and $e_{3}$ the unit normal of $M$. Then $\left(X, e_{3}\right)$ is a space-like 2-tuple in $\mathbf{R}^{2,1}$ of type $O(1,1)$, where $e_{3}$ is a parametrization of an open subset of pseudo-hyperbolic space $H^{2}(1)=\left\{q \in \mathbf{R}^{2,1} \mid\langle q, q\rangle=-1\right\}$.

Now we are interested in finding the connection between the spacelike isothermic surfaces in $\mathbf{R}^{n-j, j}$ and the solutions of the $O(n-j+1, j+1) /$ $O(n-j, j) \times O(1,1)$-system II. We begin by defining space-like isothermic surfaces in $\mathbf{R}^{n-j, j}$ just as in the classic situation of isothermic surfaces in $\mathbf{R}^{n}$ ([1]).

Definition 2.2 (Space-like isothermic surface). Let $\mathcal{O}$ be a domain in $\mathbf{R}^{2}$. An immersion $X: \mathcal{O} \rightarrow \mathbf{R}^{n-j, j}$ is called a space-like isothermic surface if it has flat normal bundle and the two fundamental forms are:

$$
I=e^{2 u}\left(d x_{1}^{2}+d x_{2}^{2}\right), \quad I I=e^{u} \sum_{i=1}^{n-2}\left(g_{i, 1} d x_{1}^{2}+g_{i, 2} d x_{2}^{2}\right) e_{i+2}
$$

with respect to some parallel normal frame $\left\{e_{\alpha}\right\}$. Or equivalently $\left(x_{1}, x_{2}\right) \in \mathcal{O}$ is conformal and line of curvature coordinate system for $X$.

It is not difficult to see that The Gauss-Codazzi-Ricci equation for space-like isothermic surfaces in $\mathbf{R}^{n-j, j}$ is (10).

Our next result establishes the relation between space-like isothermic surfaces and the space-like 2-tuples in $\mathbf{R}^{n-j, j}$ of type $O(1,1)$.

Proposition 2.1. Suppose that $\left(X_{1}, X_{2}\right)$ is a space-like 2-tuple in $\mathbf{R}^{n-j, j}$ of type $O(1,1)$. Let $Z_{1}=X_{1}-X_{2}$ and $Z_{2}=X_{1}+X_{2}$. Then both $Z_{1}$ and $Z_{2}$ are space-like isothermic.

ProOf. Let $\left(u, r_{1,1}, r_{1,2}, \ldots, r_{n-2,1}, r_{n-2,2}\right)$ be a solution of $(10)$ associated to $\left(X_{1}, X_{2}\right)$. Set $X=\left(X_{1}, X_{2}\right)$. Then by (9), we have:

$$
\left\{\begin{array}{l}
d X_{1}=-\left(\cosh u d x_{1} e_{1}+\sinh u d x_{2} e_{2}\right), \\
d X_{2}=\sinh u d x_{1} e_{1}+\cosh u d x_{2} e_{2} .
\end{array}\right.
$$


We note that $F=\left(\begin{array}{cc}0 & u_{x_{1}} \\ u_{x_{2}} & 0\end{array}\right), w_{12}=u_{x_{2}} d x_{1}-u_{x_{1}} d x_{2}$ and $w_{i \alpha}=\sigma_{\alpha} r_{\alpha-2, i} d x_{i}$ where $\sigma_{\alpha}=1, \alpha=3, \ldots, n-j$ and $\sigma_{\alpha}=-1, \alpha=n-j+1, \ldots, n$. Now using (8), we have that for $\alpha=3, \ldots, n, d e_{\alpha}=\sigma_{\alpha}\left(r_{\alpha-2,1} d x_{1} e_{1}+r_{\alpha-2,2} d x_{2} e_{2}\right)$. We compute that

$$
\left\{\begin{array}{l}
d Z_{1}=d X_{1}-d X_{2}=-e^{u}\left(d x_{1} e_{1}+d x_{2} e_{2}\right), \\
d Z_{2}=d X_{1}+d X_{2}=-e^{-u}\left(d x_{1} e_{1}-d x_{2} e_{2}\right) .
\end{array}\right.
$$

Hence the induced metric and the second fundamental form for $Z_{1}$ and $Z_{2}$ are, respectively:

$$
\left\{\begin{array}{l}
I_{1}=e^{2 u}\left(d x_{1}^{2}+d x_{2}^{2}\right) \\
I I_{1}=e^{u} \sum_{i=1}^{n-2}\left(r_{i, 1} d x_{1}^{2}+r_{i, 2} d x_{2}^{2}\right) e_{i+2} \\
I_{2}=e^{-2 u}\left(d x_{1}^{2}+d x_{2}^{2}\right) \\
I I_{2}=e^{-u} \sum_{i=1}^{n-2}\left(r_{i, 1} d x_{1}^{2}-r_{i, 2} d x_{2}^{2}\right) e_{i+2}
\end{array}\right.
$$

We observe that the two immersions $Z_{1}$ and $Z_{2}$ given in proposition above are isothermic dual surfaces, which we called a space-like isothermic pair in $\mathbf{R}^{n-j, j}$.

Now we will study the dressing action of a rational map with two simple poles on the space of solutions of the $O(n-j+1, j+1) / O(n-j, j) \times O(1,1)$ system II.

Let $O(n-j+1, j+1) \otimes \mathbf{C}=O(n-j+1, j+1 ; \mathbf{C}) \quad$ the complexified Lie group. The symmetric space $O(n-j+1, j+1) / O(n-j, j) \times O(1,1)$ is determined by two involutions, namely $\tau, \sigma: O(n-j+1, j+1 ; \mathbf{C}) \rightarrow$ $O(n-j+1, j+1 ; \mathbf{C})$ defined by: $X \mapsto \tau(X)=\bar{X}$ and $X \mapsto \sigma(X)=I_{n, 2}^{-1} X I_{n, 2}$, resp. Then $O(n-j+1, j+1) / O(n-j, j) \times O(1,1)$-reality condition is:

$$
\left\{\begin{array}{l}
\overline{g(\bar{\lambda})}=g(\lambda) \\
I_{n, 2} g(-\lambda) I_{n, 2}=g(\lambda) \\
g(\lambda)^{t}\left(\begin{array}{cc}
I_{n-j, j} & 0 \\
0 & I_{1,1}
\end{array}\right) g(\lambda)=\left(\begin{array}{cc}
I_{n-j, j} & 0 \\
0 & I_{1,1}
\end{array}\right)
\end{array}\right.
$$

for a map $g: \mathbf{C} \rightarrow U_{\mathbf{C}}=O(n-j+1, j+1 ; \mathbf{C})$.

We recall that a frame for a solution $v$ of the $U / K$-system (II) is a trivialization of the corresponding Lax connection $\theta_{\lambda}\left(\theta_{\lambda}^{I I}\right)$ that satisfies the $U / K$-reality condition.

Let 


$$
\begin{aligned}
G_{+}= & \{g: \mathbf{C} \rightarrow O(n-j+1, j+1 ; \mathbf{C}) \mid g \text { is holomorphic and satisfies the } \\
& \text { reality condition }(13)\}
\end{aligned}
$$

$$
\begin{aligned}
& G_{-}=\left\{g: S^{2} \rightarrow O(n-j+1, j+1 ; \mathbf{C}) \mid g \text { is meromorphic, } g(\infty)=I\right. \text { and } \\
&\text { satisfies the reality condition }(13)\} .
\end{aligned}
$$

Now we find certain simple elements in $G_{-}$explicitly. Let $W=$ $\left(w_{1}, \ldots, w_{n}\right)^{t} \in \mathbf{R}^{n-j, j}, Z=\left(z_{1}, z_{2}\right)^{t} \in \mathbf{R}^{1,1}$ unit vectors and $\mathbf{C}^{n+2}$ be equipped with the bi-linear form:

$$
\begin{aligned}
\langle U, V\rangle_{2}= & \bar{U}^{t}\left(\begin{array}{cc}
I_{n-j, j} & 0 \\
0 & I_{1,1}
\end{array}\right) V=\bar{u}_{1} v_{1}+\cdots+\bar{u}_{n-j} v_{n-j}-\bar{u}_{n-j+1} v_{n-j+1} \\
& -\cdots-\bar{u}_{n} v_{n}+\bar{u}_{n+1} v_{n+1}-\bar{u}_{n+2} v_{n+2} .
\end{aligned}
$$

Let $\pi$ the orthogonal projection of $\mathbf{C}^{n+2}$ onto the span of $\left(\begin{array}{c}W \\ i Z\end{array}\right)$ with respect to $\langle,\rangle_{2}$. So

$$
\pi=\frac{1}{2}\left(\begin{array}{cc}
W W^{t} & -i W Z^{t} \\
i Z W^{t} & Z Z^{t}
\end{array}\right)\left(\begin{array}{cc}
I_{n-j, j} & 0 \\
0 & I_{1,1}
\end{array}\right)
$$

$\bar{\pi}$ is the projection onto $\mathbf{C}\left(\begin{array}{c}-W \\ i Z\end{array}\right)$, which is perpendicular to $\left(\begin{array}{c}W \\ i Z\end{array}\right)$. So $\bar{\pi} \pi=\pi \bar{\pi}=0$. Let $s \in \mathbf{R}, s \neq 0$, and it defines

$$
g_{s, \pi}(\lambda)=\left(\pi+\frac{\lambda-i s}{\lambda+i s}(I-\pi)\right)\left(\bar{\pi}+\frac{\lambda+i s}{\lambda-i s}(I-\bar{\pi})\right) .
$$

So substituting (14) to $g_{s, \pi}$, we get

$$
\begin{aligned}
g_{s, \pi}(\lambda)=\frac{1}{\lambda^{2}+s^{2}} & {\left[\lambda^{2} I+s^{2}\left(\begin{array}{cc}
I-2 W W^{t} I_{n-j, j} & 0 \\
0 & I-2 Z Z^{t} I_{1,1}
\end{array}\right)\right.} \\
& \left.+2 s \lambda\left(\begin{array}{cc}
0 & W Z^{t} I_{1,1} \\
-Z W^{t} I_{n-j, j} & 0
\end{array}\right)\right] .
\end{aligned}
$$

One can see that $g_{s, \pi}(\lambda)(15)$ satisfies the $O(n-j+1, j+1) / O(n-j, j) \times$ $O(1,1)$-reality condition (13), therefore the element $g_{s, \pi} \in G_{-}$.

Now we get an explicit construction of the action of $g_{s, \pi}$ on the space of solutions of the $O(n-j+1, j+1) / O(n-j, j) \times O(1,1)$-system.

THEOREM 2.5. Let $\xi: \mathbf{R}^{2} \rightarrow \mathscr{M}_{n \times 2}$ be a solution of the $O(n-j+1, j+1) /$ $O(n-j, j) \times O(1,1)$-system (3), and $E(x, \lambda)$ a frame of $\xi$ such that $E(x, \lambda)$ is 
holomorphic for $\lambda \in \mathbf{C}$. Let $W$ and $Z$ be unit vectors in $\mathbf{R}^{n-j, j}, \mathbf{R}^{1,1}$ respectively, $\pi$ the orthogonal projection onto $\mathbf{C}\left(\begin{array}{c}W \\ i Z\end{array}\right)$ with respect to $\langle,\rangle_{2}$ and $g_{s, \pi}$ the map defined by (15). Let $\tilde{\pi}(x)$ denote the orthogonal projection onto $\mathbf{C}\left(\begin{array}{c}\tilde{W} \\ i \tilde{Z}\end{array}\right)(x)$ with respect to $\langle,\rangle_{2}$, where

$$
\left(\begin{array}{c}
\tilde{W} \\
i \tilde{Z}
\end{array}\right)(x)=E(x,-i s)^{-1}\left(\begin{array}{c}
W \\
i Z
\end{array}\right) .
$$

Let $\hat{W}=\frac{\tilde{W}}{\|\tilde{W}\|_{n-j, j}}$ and $\hat{Z}=\frac{\tilde{Z}}{\|\tilde{Z}\|_{1,1}}, \tilde{E}(x, \lambda)=g_{s, \pi}(\lambda) E(x, \lambda) g_{s, \tilde{\pi}(x)}(\lambda)^{-1}$,

$$
\tilde{\xi}=\xi-2 s\left(\hat{W} \hat{Z}^{t} I_{1,1}\right)_{*},
$$

where $\left(\vartheta_{*}\right)_{i j}=\vartheta_{i j}$ if $i \neq j$, and $\left(\vartheta_{*}\right)_{i i}=0,1 \leq i \leq 2$. Let

$$
\tilde{E}^{\#}(x, \lambda)=E(x, \lambda) g_{s, \tilde{\pi}}^{-1} .
$$

Then

(a) $\tilde{\xi}$ is a new solution of system (3).

(b) $\tilde{E}^{\#}$ is a frame for $\tilde{\xi}$.

(c)

$$
E(x, 0)=\left(\begin{array}{cc}
A(x) & 0 \\
0 & B(x)
\end{array}\right), \quad \tilde{E}^{\#}(x, 0)=\left(\begin{array}{cc}
\tilde{A}^{\#}(x) & 0 \\
0 & \tilde{B}^{\#}(x)
\end{array}\right),
$$

for some $A, B, \tilde{A}^{\#}(x), \tilde{B}^{\#}(x)$, and

$$
\tilde{A}^{\#}=A\left(I-2 \hat{W} \hat{W}^{t} I_{n-j, j}\right), \quad \tilde{B}^{\#}=B\left(I-2 \hat{Z} \hat{Z}^{t} I_{1,1}\right) .
$$

(d) $(\tilde{W}, \tilde{Z})$ is a solution of the system:

$$
d\left(\begin{array}{c}
\tilde{W} \\
i \tilde{Z}
\end{array}\right)(x)=-\theta_{-i s}\left(\begin{array}{c}
\tilde{W} \\
i \tilde{Z}
\end{array}\right)(x)
$$

where $\theta_{\lambda}$ is the Lax connection.

For proving this theorem we have the following lemma whose proof is quite similar to the proof of Lemma 9.4 in [2] and which we omit.

LEMMA 2.1. With the same conditions as in theorem above, we get

(i) $\tilde{W}(x) \in \mathbf{R}^{n}, \tilde{Z}(x) \in \mathbf{R}^{2}$.

(ii) $\|\tilde{W}(x)\|_{n-j, j}=\|\tilde{Z}(x)\|_{1,1} \forall x$ and $g_{s, \tilde{\pi}}$ satisfies the $O(n-j+1, j+1) /$ $O(n-j, j) \times O(1,1)$-reality conditions $(13)$, i.e. $g_{s, \tilde{\pi}} \in G_{-}$.

(iii) $\tilde{E}(x, \lambda)$ is holomorphic in $\lambda \in \mathbf{C}$. 
Proof OF THEOREM 2.5. The computations give us

$$
\tilde{E}^{-1} d \tilde{E}=g_{s, \tilde{\pi}} E^{-1} d E g_{s, \tilde{\pi}}^{-1}-d g_{s, \tilde{\pi}} g_{s, \tilde{\pi}}^{-1} .
$$

But $E^{-1} d E=\sum_{i=1}^{2}\left(a_{i} \lambda+\left[a_{i}, v\right]\right) d x_{i}, \quad \tilde{E}(x, \lambda) \quad$ is holomorphic in $\lambda \in \mathbf{C}$ and $g_{s, \tilde{\pi}}(\lambda)$ is holomorphic at $\lambda=\infty$. So $\tilde{E}^{-1} d \tilde{E}$ must be of the form: $\sum_{i=1}^{2}\left(a_{i} \lambda+\mu_{i}\right) d x_{i}$. Now we write

$$
g_{s, \tilde{\pi}}(\lambda)=I+\frac{m_{1}(x)}{\lambda}+\frac{m_{2}(x)}{\lambda^{2}}+\cdots
$$

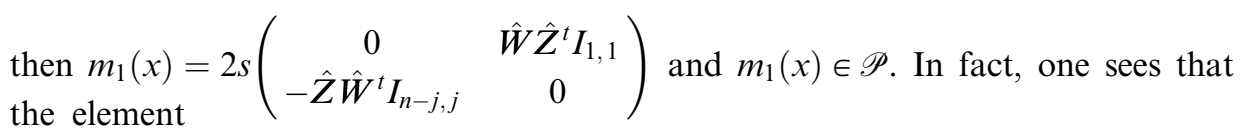

$$
\begin{aligned}
g_{s, \tilde{\pi}(x)}(\lambda) & =\left(\tilde{\pi}+\frac{\lambda-i s}{\lambda+i s}(I-\tilde{\pi})\right)\left(\overline{\tilde{\pi}}+\frac{\lambda+i s}{\lambda-i s}(I-\overline{\tilde{\pi}})\right) \\
& =\frac{\lambda+i s}{\lambda-i s} \tilde{\pi}+\frac{\lambda-i s}{\lambda+i s} \overline{\tilde{\pi}}+(I-\tilde{\pi})(I-\overline{\tilde{\pi}}),
\end{aligned}
$$

and now we make the expansion of terms $\frac{\lambda+i s}{\lambda-i s}$ and $\frac{\lambda-i s}{\lambda+i s}$ around $\lambda=\infty$. Substituting this in (21) we have

$$
\begin{aligned}
g_{s, \tilde{\pi}(x)}(\lambda)= & \left(\tilde{\pi}+\frac{2 i s}{\lambda} \tilde{\pi}+2 i s\left[\frac{i s}{\lambda^{2}}-\frac{s^{2}}{\lambda^{3}}+\cdots\right] \tilde{\pi}\right)+\left(\overline{\tilde{\pi}}-\frac{2 i s}{\lambda} \overline{\tilde{\pi}}+\frac{2 i^{2} s^{2}}{\lambda^{2}} \overline{\tilde{\pi}}+\cdots\right) \\
& +(I-\tilde{\pi})(I-\overline{\tilde{\pi}}) .
\end{aligned}
$$

Hence we obtain that $m_{1}(x)=2 i s(\tilde{\pi}-\overline{\tilde{\pi}})$, i.e., $m_{1}(x)$ is as we had claimed.

Now multiply (20) by $g_{s, \tilde{\pi}}$ on the right side and equate the constant term of equation which results of that operation, to obtain

$$
\mu_{i}=\left[a_{i}, v-m_{1}\right]=\left[a_{i}, v-p_{o}\left(m_{1}\right)\right],
$$

where $p_{o}$ is the projection from $\mathscr{P}$ onto $\mathscr{P} \cap \mathscr{A}^{\perp}$. Therefore $\tilde{v}=v-p_{o}\left(m_{1}\right)$ is a solution of $O(n-j+1, j+1) / O(n-j, j) \times O(1,1)$-system. More specifically, writing $v=\left(\begin{array}{cc}0 & \xi \\ -I_{1,1} \xi^{t} I_{n-j, j} & 0\end{array}\right)$ and $\tilde{v}=\left(\begin{array}{cc}0 & \tilde{\xi} \\ -I_{1,1} \tilde{\xi}^{t} I_{n-j, j} & 0\end{array}\right), \tilde{\xi}=\xi-2 s\left(\hat{W} \hat{Z}^{t} I_{1,1}\right)_{*}$ is a solution of $O(n-j+1, j+1) / O(n-j, j) \times O(1,1)$-system (3).

For item (b) and (c), since $g_{s, \tilde{\pi}} \in O(n-j+1, j+1 ; \mathbf{C})$, (18) becomes in 


$$
\begin{aligned}
\tilde{E}^{\#}(x, \lambda)= & E(x, \lambda)\left(\begin{array}{cc}
I_{n-j, j} & 0 \\
0 & I_{1,1}
\end{array}\right) \frac{1}{\lambda^{2}+s^{2}} \\
\times & {\left[\lambda^{2} I+s^{2}\left(\begin{array}{cc}
I-2 I_{n-j, j} \hat{W} \hat{W}^{t} & 0 \\
0 & I-2 I_{1,1} \hat{Z} \hat{Z}^{t}
\end{array}\right)\right.} \\
& \left.+2 s \lambda\left(\begin{array}{cc}
0 & -I_{n-j, j} \hat{W} \hat{Z}^{t} \\
I_{1,1} \hat{Z} \hat{W}^{t} & 0
\end{array}\right)\right]\left(\begin{array}{cc}
I_{n-j, j} & 0 \\
0 & I_{1,1}
\end{array}\right) .
\end{aligned}
$$

We note that $\tilde{E}^{\#}$ is a frame of $\tilde{\xi}$ and that $\tilde{E}^{\#}(x,$.$) is not in G_{+}$. The reality condition (13) implies that both $E(x, 0)$ and $\tilde{E}^{\#}(x, 0)$ are in $O(n-j, j) \times O(1,1)$. So, now we write:

$$
E(x, 0)=\left(\begin{array}{cc}
A(x) & 0 \\
0 & B(x)
\end{array}\right), \quad \tilde{E}^{\#}(x, 0)=\left(\begin{array}{cc}
\tilde{A}^{\#}(x) & 0 \\
0 & \tilde{B}^{\#}(x)
\end{array}\right) .
$$

Finally, from $(18)$ we have $\tilde{E}^{\#}(x, 0)=E(x, 0) g_{s, \tilde{\pi}(x)}(0)^{-1}$, this means

$$
\begin{aligned}
\left(\begin{array}{cc}
\tilde{A}^{\#} & 0 \\
0 & \tilde{B}^{\#}
\end{array}\right)= & \left(\begin{array}{cc}
A & 0 \\
0 & B
\end{array}\right)\left(\begin{array}{cc}
I_{n-j, j} & 0 \\
0 & I_{1,1}
\end{array}\right)\left(\begin{array}{cc}
I-2 I_{n-j, j} \hat{W} \hat{W}^{t} & 0 \\
0 & I-2 I_{1,1} \hat{Z} \hat{Z}^{t}
\end{array}\right) \\
& \times\left(\begin{array}{cc}
I_{n-j, j} & 0 \\
0 & I_{1,1}
\end{array}\right) \\
= & \left(\begin{array}{cc}
A\left(I-2 \hat{W} \hat{W}^{t} I_{n-j, j}\right) & 0 \\
0 & B\left(I-2 \hat{Z} \hat{Z}^{t} I_{1,1}\right)
\end{array}\right),
\end{aligned}
$$

from which follows $\tilde{A}^{\#}=A\left(I-2 \hat{W} \hat{W}^{t} I_{n-j, j}\right), \tilde{B}^{\#}=B\left(I-2 \hat{Z} \hat{Z}^{t} I_{1,1}\right)$.

(d) Follows directly taking the differential of (16).

In the next statement we will use the notation $g_{s, \pi} \# \xi$, for denoting the dressing action of $g_{s, \pi}$ on $\xi$, and $\tilde{g}_{-} \# \xi$ for the dressing action of $\tilde{g}_{-}$on $\xi$ $($ see $([2]))$.

Corollary 2.1. Suppose $E$ is a frame of the solution $\xi$ of the $O(n-j+1, j+1) / O(n-j, j) \times O(1,1)$-system (3) such that $E(x, \lambda)$ is holomorphic for $\lambda \in \mathbf{C}$.

(i) If $E(0, \lambda)=I$, then $\tilde{\xi}$ obtained in Theorem 2.5, is $g_{s, \pi} \# \xi$ and $\tilde{E}$ is the frame of $\tilde{\xi}$ with $\tilde{E}(0, \lambda)=I$.

(ii) Let $g_{+}(\lambda)=E(0, \lambda)$ and $\tilde{\xi}$ the new solution of (3) obtained in Theorem 2.5. Then $g_{+} \in G_{+}$and $\tilde{\xi}=\tilde{g}_{-} \# \xi$, where $\tilde{g}_{-}$is obtained by factoring $g_{s, \pi} g_{+}=\tilde{g}_{+} \tilde{g}_{-}$ with $\tilde{g}_{ \pm} \in G_{ \pm}$. 
Now writing $\xi=\left(\begin{array}{c}F \\ G\end{array}\right), \tilde{\xi}=\left(\begin{array}{c}\tilde{F} \\ \tilde{G}\end{array}\right)$, we rewrite the new solution $\tilde{\xi}$ given by Theorem 2.5 as

$$
\left(\begin{array}{c}
\tilde{F} \\
\tilde{G}
\end{array}\right)=\left(\begin{array}{c}
F \\
G
\end{array}\right)-2 s\left(\hat{W} \hat{Z}^{t} I_{1,1}\right)_{*} .
$$

So $(F, G, B)$ and $\left(\tilde{F}, \tilde{G}, \tilde{B}^{\#}\right)$ are solutions of the $O(n-j+1, j+1) / O(n-j, j) \times$ $O(1,1)$-system II (4).

We note that if we write $F=\left(f_{i j}\right)_{2 \times 2}, \quad G=\left(r_{i j}\right)_{(n-2) \times 2}, \quad \tilde{F}=\left(\tilde{f}_{i j}\right)_{2 \times 2}, \quad \tilde{G}=$ $\left(\tilde{r}_{i j}\right)_{(n-2) \times 2}$, then $(23)$ for $\tilde{\xi}$ is

$$
\left\{\begin{array}{l}
\tilde{f}_{i j}=f_{i j}-2 s \hat{w}_{i} \hat{z}_{j} \varepsilon_{j}, \quad i, j=1,2 \\
\tilde{r}_{i j}=r_{i j}-2 s \hat{w}_{2+i} \hat{z}_{j} \varepsilon_{j}, \quad i=1, \ldots, n-2, j=1,2
\end{array}\right.
$$

where $\varepsilon_{1}=-\varepsilon_{2}=1$.

Now let $\tilde{E}^{\#}$ frame of $\tilde{\xi}, E^{I I}$ of $(F, G, B)$ and $\widetilde{E^{\#}}{ }^{I I}$ of $\left(\tilde{F}, \tilde{G}, \widetilde{B^{\#}}\right)$. Then we get

$$
\widetilde{E^{\#} I I}(x, \lambda)=\widetilde{E^{\#}}(x, \lambda)\left(\begin{array}{cc}
I_{n-j, j} & 0 \\
0 & I_{1,1}
\end{array}\right)\left(\begin{array}{cc}
I & 0 \\
0 & \widetilde{B^{\#}}
\end{array}\right)\left(\begin{array}{cc}
I_{n-j, j} & 0 \\
0 & I_{1,1}
\end{array}\right),
$$

now, using (22), we get that the frames $E^{I I}$ and $\widetilde{E^{\#}}{ }^{I I}$ are related by

$$
\widetilde{E^{\#} I I}(x, \lambda)=E^{I I}(x, \lambda)\left[I-\frac{2}{\lambda^{2}+s^{2}}\left(\begin{array}{cc}
s^{2} \hat{W} \hat{W}^{t} I_{n-j, j} & -s \lambda \hat{W} \hat{Z}^{t} B^{t} I_{1,1} \\
-s \lambda B \hat{Z} \hat{W}^{t} I_{n-j, j} & \lambda^{2} B \hat{Z} \hat{Z}^{t} B^{t} I_{1,1}
\end{array}\right)\right] .
$$

In the next we will use the notation

$$
\begin{gathered}
\left(\tilde{\xi}, \tilde{E}^{\#}\right)=g_{s, \pi} \cdot(\xi, E), \quad \tilde{A}^{\#}=g_{s, \pi} \cdot A, \quad \tilde{B}^{\#}=g_{s, \pi} \cdot B, \\
\left(\tilde{F}, \tilde{G}, \tilde{B}^{\#}, \widetilde{E^{\# I I}}\right)=g_{s, \pi} \cdot\left(F, G, B, E^{I I}\right),
\end{gathered}
$$

to understand the result obtained after the action of the element $g_{s, \pi}$ over the solutions given.

\section{Associated Geometric Transformations}

Here we describe the corresponding geometric transformations on surfaces in the pseudo-riemannian space $\mathbf{R}^{n-j, j}$ corresponding to the action of $g_{s, \pi}$, given in (15), on the space of local solution of the $O(n-j+1, j+1) / O(n-j, j) \times$ $O(1,1)$-system II (4).

We start with the definition of Ribaucour transformation given by DajczerTojeiro in [6]. Let $\mathbf{R}_{s}^{n+p}$ be the standard flat pseudo-riemannian space form of index $s, 0 \leq s \leq p$. For $x \in \mathbf{R}_{s}^{n+p}$ and $v \in\left(T \mathbf{R}_{s}^{n+p}\right)_{x}$, where let $\gamma_{x, v}(t)=\exp (t v)$ denote the geodesic. 
Definition 3.1. Let $M^{n}$ and $\tilde{M}^{n}$ be riemannian submanifolds of the pseudoriemannian space $\mathbf{R}_{s}^{n+p}, 0 \leq s \leq p$. A sphere congruence is a vector bundle isomorphism $P: \mathscr{V}(M) \rightarrow \mathscr{V}(\tilde{M})$ that covers a diffeomorphism $l: M \rightarrow \tilde{M}$ with the following conditions:

(1) If $\xi$ is a parallel normal vector field of $M$, then $P \circ \xi \circ l^{-1}$ is a parallel normal field of $\tilde{M}$.

(2) For any nonnull vector $\xi \in \mathscr{V}_{x}(M)$, the geodesics $\gamma_{x, \xi}$ and $\gamma_{l(x), P(\xi)}$ intersect at a point that is equidistant from $x$ and $l(x)$ (the distance depends on $x$ ).

Definition 3.2. A sphere congruence $P: \mathscr{V}(M) \rightarrow \mathscr{V}(\tilde{M})$ that covers $l: M \rightarrow \tilde{M}$ is called a Ribaucour transformation if it satisfies the following additional properties:

(1) If $e$ is an eigenvector of the shape operator $A_{\xi}$ of $M$, then $l_{*}(e)$ is an eigenvector of the shape operator $A_{P(\xi)}$ of $\tilde{M}$.

(2) The geodesics $\gamma_{x, e}$ and $\gamma_{l(x), l_{*}(e)}$ intersect at a point that is equidistant to $x$ and $l(x)$.

THEOREM 3.1. Let $\xi=\left(\begin{array}{l}F \\ G\end{array}\right)$ solution of (3), E frame of $\xi, E(x, 0)=$ $\left(\begin{array}{cc}A(x) & 0 \\ 0 & B(x)\end{array}\right), \quad(F, G, B) \quad$ a solution corresponding to $O(n-j+1, j+1) /$ $O(n-j, j) \times O(1,1)$-system $I I(4)$, and

$$
\left(\tilde{F}, \tilde{G}, \tilde{B}^{\#}, \widetilde{E^{\#}} I I\right)=g_{s, \pi} \cdot\left(F, G, B, E^{I I}\right), \quad \tilde{A}^{\#}=g_{s, \pi} \cdot A .
$$

Let $e_{i}, \widetilde{e}_{i}$ denote the $i$-th column of $A$ and $\tilde{A}^{\#}$ resp. Then we have

(i)

$$
\begin{aligned}
& \frac{\partial E}{\partial \lambda}(x, 0) E^{-1}(x, 0)=\left(\begin{array}{cc}
0 & X \\
-I_{1,1} X^{t} I_{n-j, j} & 0
\end{array}\right), \\
& \frac{\partial \widetilde{E^{\#}}}{\partial \lambda}(x, 0) \widetilde{E^{\#}-1}(x, 0)=\left(\begin{array}{cc}
0 & \tilde{X} \\
-I_{1,1} \tilde{X}^{t} I_{n-j, j} & 0
\end{array}\right)
\end{aligned}
$$

for some $X$ and $\tilde{X}$.

(ii) $X=\left(X_{1}, X_{2}\right)$ and $\tilde{X}=\left(\tilde{X}_{1}, \tilde{X}_{2}\right)$ are space-like 2-tuples in $\mathbf{R}^{n-j, j}$ of type $O(1,1)$ such that $\left\{e_{\alpha}\right\}$ and $\left\{\tilde{e}_{\alpha}\right\}$ are resp. parallel normal frames for $X_{j}$ and $\tilde{X}_{j}$ for $j=1,2$, with indices $\alpha=3, \ldots, n-j$ and $\alpha=n-j+1, \ldots, n$ corresponding to space-like and time-like vectors resp.

(iii) The solutions of $O(n-j+1, j+1) / O(n-j, j) \times O(1,1)$-system II (4) corresponding to $X$ and $\tilde{X}$ as given in Theorem 2.2 are $(F, G, B)$ and $\left(\tilde{F}, \tilde{G}, \tilde{B}^{\#}\right)$ resp. 
(iv) The bundle morphism $P\left(e_{k}(x)\right)=\tilde{e}_{k}(x), k=3, \ldots, n$ is a Ribaucour Transformation covering the map $X_{j}(x) \mapsto \tilde{X}_{j}(x)$ for each $1 \leq j \leq 2$.

(v) There exist smooth functions $\phi_{i j}$ such that $X_{j}+\phi_{i j} e_{i}=\tilde{X}_{j}+\phi_{i j} \tilde{e}_{i}$ for $1 \leq j \leq 2$ and $1 \leq i \leq n$.

For the proof we will need the following result whose proof is quite similar to proof of Corollary (6.11) in [2].

Proposition 3.1. Let $E(x, \lambda)$ be a frame for the solution $\xi$ of system (3), and $Y(x)=\frac{\partial E}{\partial \lambda}(x, 0) E^{-1}(x, 0)$. Then we have

(i)

$$
Y=\left(\begin{array}{cc}
0 & X \\
-I_{1,1} X^{t} I_{n-j, j} & 0
\end{array}\right) \text { for some } X \in \mathscr{M}_{n \times 2} .
$$

(ii) $X=\left(X_{1}, X_{2}\right)$ is a space-like 2-tuple in $\mathbf{R}^{n-j, j}$ of type $O(1,1)$.

(iii) $d X=A\left(\begin{array}{ccccc}-d x_{1} & 0 & 0 & \cdots & 0 \\ 0 & d x_{2} & 0 & \cdots & 0\end{array}\right)^{t} B^{-1}$. This means $X$ satisfies (9).

Proof of Theorem 3.1. From (18), Theorem 2.1, proposition above, we get

$$
\begin{aligned}
& \left(\begin{array}{cc}
0 & \tilde{X} \\
-I_{1,1} \tilde{X}^{t} I_{n-j, j} & 0
\end{array}\right)=\frac{\partial \widetilde{E^{\#}}}{\partial \lambda}(x, 0) \widetilde{E^{\#}}-1(x, 0) \\
& =\frac{\partial E}{\partial \lambda}(x, 0) E^{-1}(x, 0)+\frac{2}{s} E(x, 0)\left(\begin{array}{cc}
0 & \hat{W} \hat{Z}^{t} I_{1,1} \\
-\hat{Z} \hat{W}^{t} I_{n-j, j} & 0
\end{array}\right) E(x, 0)^{-1} \\
& =\left(\begin{array}{cc}
0 & X \\
-I_{1,1} X^{t} I_{n-j, j} & 0
\end{array}\right)+\frac{2}{S}\left(\begin{array}{cc}
0 & A \hat{W} \hat{Z}^{t} B^{t} I_{1,1} \\
-B \hat{Z} \hat{W}^{t} A^{t} I_{n-j, j} & 0
\end{array}\right) .
\end{aligned}
$$

Hence

$$
\tilde{X}=X+\frac{2}{S} A \hat{W} \hat{Z}^{t} B^{t} I_{1,1} .
$$

Let now $\eta=\sum_{j=1}^{n} \hat{w}_{j} e_{j}$, then the $i$-th column of $\tilde{X}$ is given by

$$
\widetilde{X}_{i}=X_{i}+\frac{2}{S} \varepsilon_{i} \sum_{j=1}^{2}\left(\hat{z}_{j} b_{i j}\right) \eta
$$

where $\varepsilon_{1}=-\varepsilon_{2}=1$. Now from the relation $\tilde{A}^{\#}=A\left(I-2 \hat{W} \hat{W}^{t} I_{n-j, j}\right)$ we get $\tilde{e}_{i}=e_{i}-2 \hat{w}_{i} \eta \varepsilon_{i}$ with $\varepsilon_{i}=1, i=1, \ldots, n-j$ and $\varepsilon_{i}=-1, i=n-j+1, \ldots, n$. So using this last relation, we have 


$$
X_{j}+\phi_{i j} e_{i}=\tilde{X}_{j}+\phi_{i j} \tilde{e}_{i}
$$

where

$$
\phi_{i j}=\frac{\varepsilon_{i} \varepsilon_{j}}{s \hat{w}_{i}} \sum_{k=1}^{2} \hat{z}_{k} b_{j k}, \quad \text { for } j=1,2, i=1, \ldots, n
$$

This proves that $P: \mathscr{V}\left(X_{j}\right) \rightarrow \mathscr{V}\left(\tilde{X}_{j}\right)$ given by $P\left(e_{k}(x)\right)=\tilde{e}_{k}(x), k=3, \ldots, n$ is a Ribaucour transformation covering the map $l: X_{j} \rightarrow \tilde{X}_{j}, l\left(X_{j}(x)\right)=\tilde{X}_{j}(x)$ for each $1 \leq j \leq 2$.

The next result shows that the transformation constructed in Theorem 3.1, for space-like 2-tuples in $\mathbf{R}^{n-j, j}$ of type $O(1,1)$, is a Darboux transformation for spacelike isothermic surfaces.

Definition 3.3. Let $M, \tilde{M}$ be two space-like surfaces in $\mathbf{R}^{n-j, j}$ with flat and non-degenerate normal bundle and $P: \mathscr{V}(M) \rightarrow \mathscr{V}(\tilde{M})$ a Ribaucour transformation that covers the map $l: M \rightarrow \tilde{M}$. If $l$ is a conformal diffeomorphism, then $P$ is called a Darboux transformation.

THEOREM 3.2. Let $\left(Y_{1}, Y_{2}\right)$ be a space-like isothermic pair in $\mathbf{R}^{n-j, j}$ corresponding to the solution $(u, G)$ of the system $(10)$, and let $\xi=\left(\begin{array}{l}F \\ G\end{array}\right)$ the corresponding solution of the system (3), where $F=\left(\begin{array}{cc}0 & u_{x_{1}} \\ u_{x_{2}} & 0\end{array}\right)$. Let also $s \in \mathbf{R}$ different of zero, $\pi$ a projection on $\mathbf{C}\left(\begin{array}{c}W \\ i Z\end{array}\right), g_{s, \pi}$ the rational element defined in $(15)$, and $\hat{W}, \hat{Z}$ as in Theorem 2.5, for the solution $\xi$ of the system (3). Let

$$
B=\left(\begin{array}{cc}
\cosh u & \sinh u \\
\sinh u & \cosh u
\end{array}\right), \quad\left(\widetilde{E^{\#} I I}, \tilde{A}^{\#}, \tilde{B}^{\#}\right)=g_{s, \pi} \cdot\left(E^{I I}, A, B\right)
$$

Write $A=\left(e_{1}, \ldots, e_{n}\right)$ and $\tilde{A}^{\#}=\left(\tilde{e}_{1}, \ldots, \tilde{e}_{n}\right)$. Set

$$
\left\{\begin{array}{l}
\tilde{Y}_{1}=Y_{1}+\frac{2}{s}\left(\hat{z}_{1}+\hat{z}_{2}\right) e^{u} \sum_{i=1}^{n} \hat{w}_{i} e_{i} \\
\tilde{Y}_{2}=Y_{2}+\frac{2}{s}\left(\hat{z}_{1}-\hat{z}_{2}\right) e^{-u} \sum_{i=1}^{n} \hat{w}_{i} e_{i}
\end{array}\right.
$$

Then

(i) $\left(\tilde{Y}_{1}, \tilde{Y}_{2}\right)$ is also a space-like isothermic pair in $\mathbf{R}^{n-j, j}$.

(ii) $(\tilde{u}, \tilde{G})$ is the solution of system (10), corresponding to $\left(\tilde{Y}_{1}, \tilde{Y}_{2}\right)$, where $\tilde{u}=2 \alpha-u$, sinh $\alpha=-\hat{z}_{2}$ and $\tilde{G}=\left(\tilde{r}_{i j}\right)$ is defined by (24). 
(iii) The fundamental forms of pair $\left(\tilde{Y}_{1}, \tilde{Y}_{2}\right)$ are respectively

$$
\left\{\begin{array}{l}
\tilde{I}_{1}=e^{-2 \tilde{u}}\left(d x_{1}^{2}+d x_{2}^{2}\right) \\
\widetilde{I I}_{1}=e^{-\tilde{u}} \sum_{\alpha=3}^{n}\left(-\tilde{r}_{\alpha-2,1} d x_{1}^{2}+\tilde{r}_{\alpha-2,2} d x_{2}^{2}\right) \tilde{e}_{\alpha} \\
\tilde{I}_{2}=e^{2 \tilde{u}}\left(d x_{1}^{2}+d x_{2}^{2}\right) \\
\widetilde{I I}_{2}=-e^{\tilde{u}} \sum_{\alpha=3}^{n}\left(\tilde{r}_{\alpha-2,1} d x_{1}^{2}+\tilde{r}_{\alpha-2,2} d x_{2}^{2}\right) \tilde{e}_{\alpha} .
\end{array}\right.
$$

(iv) The bundle morphism $P\left(e_{k}(x)\right)=\tilde{e}_{k}(x), k=3, \ldots, n$ covering the map $Y_{i} \rightarrow \tilde{Y}_{i}$ is a Darboux transformation for each $i=1,2$.

Proof. It follows from Proposition 2.1 and Theorem 2.3 that $(F, G, B)$ is a solution of $O(n-j+1, j+1) / O(n-j, j) \times O(1,1)$-system II (5), and $X=$ $\left(X_{1}, X_{2}\right)=\left(\frac{Y_{1}+Y_{2}}{2}, \frac{Y_{2}-Y_{1}}{2}\right)$ is the corresponding space-like 2-tuple in $\mathbf{R}^{n-j, j}$ of type $O(1,1)$. Now let $\tilde{X}=\left(\tilde{X}_{1}, \tilde{X}_{2}\right)$ be as in Theorem 3.1 and let $\tilde{Y}_{1}=\tilde{X}_{1}-\tilde{X}_{2}$, $\tilde{Y}_{2}=\tilde{X}_{1}+\tilde{X}_{2}$. Then using (26) we get that $\tilde{Y}_{1}$ and $\tilde{Y}_{2}$ are given by (28). Since $\hat{z}_{1}^{2}-\hat{z}_{2}^{2}=1$, there exists a function $\alpha: \mathbf{R}^{2} \rightarrow \mathbf{R}$ such that $\hat{z}_{1}=\cosh \alpha, \hat{z}_{2}=$ $-\sinh \alpha$. It follows from $\tilde{B}^{\#}=B\left(I-2 \hat{Z} \hat{Z}^{t} I_{1,1}\right)$ that

$$
\tilde{B}^{\#}=\left(\begin{array}{cc}
-\cosh (2 \alpha-u) & -\sinh (2 \alpha-u) \\
\sinh (2 \alpha-u) & \cosh (2 \alpha-u)
\end{array}\right)=\left(\begin{array}{cc}
-\cosh \tilde{u} & -\sinh \tilde{u} \\
\sinh \tilde{u} & \cosh \tilde{u}
\end{array}\right) .
$$

Since $d \tilde{X}_{1}=\cosh \tilde{u} d x_{1} \tilde{e}_{1}+\sinh \tilde{u} d x_{2} \tilde{e}_{2}$, and $d \tilde{X}_{2}=\sinh \tilde{u} d x_{1} \tilde{e}_{1}+\cosh \tilde{u} d x_{2} \tilde{e}_{2}$, it follows that

$$
d \tilde{Y}_{1}=d \tilde{X}_{1}-d \tilde{X}_{2}=e^{-\tilde{u}}\left(d x_{1} \tilde{e}_{1}-d x_{2} \tilde{e}_{2}\right), \quad d \tilde{Y}_{2}=d \tilde{X}_{1}+d \tilde{X}_{2}=e^{\tilde{u}}\left(d x_{1} \tilde{e}_{1}+d x_{2} \tilde{e}_{2}\right) .
$$

So we get the claim (i)-(iii).

For (iv) we observe that the map $l: Y_{i} \rightarrow \tilde{Y}_{i}$ is conformal because the coordinates $\left(x_{1}, x_{2}\right)$ are isothermic for $Y_{i}$ and $\tilde{Y}_{i}$. Now we need to prove that $P: \mathscr{V}\left(Y_{i}\right) \rightarrow \mathscr{V}\left(\tilde{Y}_{i}\right)$ given by $e_{k}(x) \rightarrow \tilde{e}_{k}(x), k=3, \ldots, n$ is a Ribaucour transformation. For that, we use the fact that there exist smooth functions $\phi_{i j}$ such that $X_{j}+\phi_{i j} e_{i}=\tilde{X}_{j}+\phi_{i j} \tilde{e}_{i}$ for $1 \leq i \leq n$ and $j=1,2$, (Theorem $3.1(\mathrm{v})$ ), and so

$$
Y_{1}+\left(\phi_{i 1}-\phi_{i 2}\right) e_{i}=\tilde{Y}_{1}+\left(\phi_{i 1}-\phi_{i 2}\right) \tilde{e}_{i}, \quad Y_{2}+\left(\phi_{i 1}+\phi_{i 2}\right) e_{i}=\tilde{Y}_{2}+\left(\phi_{i 1}+\phi_{i 2}\right) \tilde{e}_{i},
$$

for each $1 \leq i \leq n$. Hence the map $P$ is a Darboux transformation.

EXAMPLE 3.1. Let $n=3, j=1$, then we have the $O(3,2) / O(2,1) \times O(1,1)$ system. Let $\left(u, r_{11}, r_{12}\right)=(0,0,0)$ be a trivial solution of $(10)$, then $F=0, G=0$, $B=I$. So a space-like 2-tuple $X$ in $\mathbf{R}^{2,1}$ of type $O(1,1)$ and the frame $E(x, y, \lambda)$ associated to trivial solution are: 


$$
X=\left(\begin{array}{cc}
-x & 0 \\
0 & y \\
0 & 0
\end{array}\right), \quad E(x, y, \lambda)=\left(\begin{array}{ccccc}
\cos (\lambda x) & 0 & 0 & -\sin (\lambda x) & 0 \\
0 & \cosh (\lambda y) & 0 & 0 & \sinh (\lambda y) \\
0 & 0 & 1 & 0 & 0 \\
\sin (\lambda x) & 0 & 0 & \cos (\lambda x) & 0 \\
0 & \sinh (\lambda y) & 0 & 0 & \cosh (\lambda y)
\end{array}\right)
$$

Then from (16), we obtain that

$$
\left(\begin{array}{c}
\tilde{w}_{1} \\
\tilde{w}_{2} \\
\tilde{w}_{3} \\
\tilde{z}_{1} \\
\tilde{z}_{2}
\end{array}\right)=\left(\begin{array}{c}
w_{1} \cosh s x+z_{1} \sinh s x \\
w_{2} \cos s y-z_{2} \sin s y \\
w_{3} \\
w_{1} \sinh s x+z_{1} \cosh s x \\
w_{2} \sin s y+z_{2} \cos s y
\end{array}\right)
$$

From (26), we get that the 2-tuple in $\mathbf{R}^{2,1}$ of type $O(1,1)$ constructed by applying the Ribaucour transformation to the trivial solution is:

$$
\tilde{X}_{1}=X_{1}+\frac{2}{s} \hat{z}_{1} \sum_{i=1}^{3} \hat{w}_{i} e_{i}, \quad \tilde{X}_{2}=X_{2}-\frac{2}{s} \hat{z}_{2} \sum_{i=1}^{3} \hat{w}_{i} e_{i}
$$

i.e.,

$$
\begin{aligned}
\tilde{X}_{1}= & \left(\begin{array}{c}
-x \\
0 \\
0
\end{array}\right)+\frac{2}{s} \frac{w_{1} \sinh s x+z_{1} \cosh s x}{\left(w_{1} \sinh s x+z_{1} \cosh s x\right)^{2}-\left(w_{2} \sin s y+z_{2} \cos s y\right)^{2}} \\
& \times\left(\begin{array}{c}
w_{1} \cosh s x+z_{1} \sinh s x \\
w_{2} \cos s y-z_{2} \sin s y
\end{array}\right) \\
\tilde{X}_{2}= & \left(\begin{array}{c}
0 \\
y \\
0
\end{array}\right)-\frac{w_{3}}{s} \frac{w_{2} \sin s y+z_{2} \cos s y}{\left(w_{1} \sinh s x+z_{1} \cosh s x\right)^{2}-\left(w_{2} \sin s y+z_{2} \cos s y\right)^{2}} \\
& \times\left(\begin{array}{c}
w_{1} \cosh s x+z_{1} \sinh s x \\
w_{2} \cos s y-z_{2} \sin s y \\
w_{3}
\end{array}\right) .
\end{aligned}
$$

EXAMPlE 3.2. A space-like plane in $\mathbf{R}^{2,1}$ is an isothermic surface corresponding to trivial solution $(0,0,0)$ of $(10)$. Then the space-like isothermic pair associated to the trivial solution is: 


$$
Y_{1}=\left(\begin{array}{c}
-x \\
-y \\
0
\end{array}\right), \quad Y_{2}=\left(\begin{array}{c}
-x \\
y \\
0
\end{array}\right)
$$

So the isothermic pair obtained by applying the Darboux transformations to the trivial solution, given by (28) is:

$$
\tilde{Y}_{1}=Y_{1}+\frac{2}{s}\left(\hat{z}_{1}+\hat{z}_{2}\right) \sum_{i=1}^{3} \hat{w}_{i} e_{i}, \quad \tilde{Y}_{2}=Y_{2}+\frac{2}{s}\left(\hat{z}_{1}-\hat{z}_{2}\right) \sum_{i=1}^{3} \hat{w}_{i} e_{i},
$$

i.e.,

$$
\begin{aligned}
\tilde{Y}_{1}= & \left(\begin{array}{c}
-x \\
-y \\
0
\end{array}\right)+\frac{2}{s} \frac{w_{1} \sinh s x+z_{1} \cosh s x+w_{2} \sin s y+z_{2} \cos s y}{\left(w_{1} \sinh s x+z_{1} \cosh s x\right)^{2}-\left(w_{2} \sin s y+z_{2} \cos s y\right)^{2}} \\
& \times\left(\begin{array}{c}
w_{1} \cosh s x+z_{1} \sinh s x \\
w_{2} \cos s y-z_{2} \sin s y \\
w_{3}
\end{array}\right) \\
\tilde{Y}_{2}= & \left(\begin{array}{c}
-x \\
y \\
0
\end{array}\right)+\frac{2}{s} \frac{w_{1} \sinh s x+z_{1} \cosh s x-w_{2} \sin s y-z_{2} \cos s y}{\left(w_{1} \sinh s x+z_{1} \cosh s x\right)^{2}-\left(w_{2} \sin s y+z_{2} \cos s y\right)^{2}} \\
& \times\left(\begin{array}{c}
w_{1} \cosh s x+z_{1} \sinh s x \\
w_{2} \cos s y-z_{2} \sin s y \\
w_{3}
\end{array}\right)
\end{aligned}
$$

\section{Appendix: Moving Frames}

We review the method of moving frames for space-like surfaces in the Lorentz space $\mathbf{R}^{n-j, j}$ : Set

$$
e_{A} \cdot e_{B}=\sigma_{A B}=I_{n-j, j}=\left(\begin{array}{cc}
I_{n-j} & 0 \\
0 & -I_{j}
\end{array}\right) \text {. }
$$

We also let $\sigma_{i}:=\sigma_{i i}$. For the space-like immersion $X$ set $d X=\omega_{1} e_{1}+\omega_{2} e_{2}$, with $e_{1}, e_{2}$ space-like unit tangent vectors to the surface and the normal space is spanned by $e_{\beta}$, for $3 \leq \beta \leq n$. Define

$$
d e_{B}=\sum_{A} \omega_{A B} e_{A}
$$

This gives $\omega_{A B}=\sigma_{A} e_{A} \cdot d e_{B}$ and $\omega_{A B} \sigma_{A}+\omega_{B A} \sigma_{B}=0$. 
From $d(d X)=0$ we get:

$$
d \omega_{1}=\omega_{2} \wedge \omega_{12}, \quad d \omega_{2}=\omega_{1} \wedge \omega_{21}, \quad \omega_{1} \wedge \omega_{\beta 1}+\omega_{2} \wedge \omega_{\beta 2}=0,
$$

for $\beta$ as above.

In addition, by Cartan's Lemma we have:

$$
\omega_{1 \beta}=h_{11}^{\beta} \omega_{1}+h_{12}^{\beta} \omega_{2}, \quad \omega_{2 \beta}=h_{21}^{\beta} \omega_{1}+h_{22}^{\beta} \omega_{2},
$$

this makes the first and second fundamental form:

$$
I: \omega_{1}^{2}+\omega_{2}^{2}, \quad I I:-\sum_{k=1,2} \sum_{\alpha} \omega_{k \alpha} \omega_{k} \sigma_{\alpha} e_{\alpha}
$$

We also have: $d \omega_{C A}=-\sum_{B} \omega_{C B} \wedge \omega_{B A}$, which yield the Gauss and Codazzi equations. The Gauss equation comes from examining $d \omega_{12}$, while the Codazzi equations are from $d \omega_{1 \beta}$ and $d \omega_{2 \beta}$.

\section{References}

[ 1 ] Burstall, F., Isothermic surfaces: conformal geometry, Clifford algebras and integrable systems, preprint, math-dg/0003096.

[2 ] Brück, M., Du, X., Park, J., Terng, C.-L., The Submanifolds Geometries associated to Grassmannian Systems, Memoirs of A.M.S. no. 735 (2002).

[ 3 ] Burstall, F., Hertrich, J. U., Pedit, F., Pinkall, U., Curved flats and isothermic surfaces, Math. Z. no. 2, 225 (1997).

[ 4 ] Cieslinski, J., Goldstein, P., Sym, A., Isothermic surfaces in $\mathbf{R}^{3}$ as soliton surfaces, Phys. Lett. A. 205 (1995), 37-43.

[ 5 ] Cieslinski, J., The Darboux-Bianchi transformation for isothermic surfaces, Isothermic surfaces, Diff. Geom. Appl. 7 (1997), 1-28.

[6] Dajczer, M., Tojeiro, R., Commuting Codazzi tensors and the Ribaucour transformation for submanifolds, Results in Mathematics. 44 (2003), 258-278.

[ 7 ] Dussan, M. P., Geometry of surfaces corresponding to $O(3,2) / O(2,1) \times O(1,1)$-system, Relatorio de Pesquisa, Outubro, 60 (2002). Universidade Estadual de Campinas, Brazil.

[ 8 ] Fujioka, A., Inoguchi, J., Spacelike surfaces and Harmonic Inverse Mean Curvature, J. Math. sci. Univ. Tokyo. 7 (2000), 657-698.

[ 9 ] Terng, C.-L., Solitons equations and Differential Geometry, J. Differential Geom. 45 (1997), no. 2, 407-445.

[10] Terng, C.-L., Uhlenbeck, K., Backlund transformations and loop group actions, Comm. Pure. Appl. Math. 53 (2000), 1-75.

[11] Tian, C., Backlund transformation on surfaces with $K=-1$ in $\mathbf{R}^{2,1}$, J. Geometry and Physics, 22 (1997), 212-218.

Author's address: IME-USP, C.P. 66281

CEP 05315-970, São Paulo, SP. Brazil 